\title{
Research on the Intelligent Hardware Influence on Design Education and
}

\section{Teaching Mode}

\author{
Wei Sun ${ }^{1 . a}$ and Yingchun Hou ${ }^{2 . b}$
}

${ }^{1}$ School of Digital Media \& Design Arts, Beijing University of Posts and Telecommunications, Beijing 100876,

China

${ }^{2}$ School of space, Space Engineering University, Beijing 101416,China.

asunweycang@163.com

\begin{abstract}
With the rapid development of various open source hardware platforms, intelligent hardware has been more and more introduced into the practice teaching of design education. This paper first describes the characteristics of intelligent hardware and the change of design ecological model, and then analyzes the impact of intelligent hardware on design education. At last, this paper illustrates how to build a problem oriented intelligent hardware teaching model through 4 problem guidance models.
\end{abstract}

Keywords: Intelligent Hardware; Design Education; Teaching Mode

\section{智能硬件对设计教育影响与教学模式探究}

\author{
孙炜 $^{1}$, 侯迎春 ${ }^{2}$ \\ ${ }^{1}$ 北京邮电大学、数字媒体与设计艺术学院、北京 100876 \\ ²航天工程大学、北京、101416
}

摘要: 随着各种开源硬件平台的迅速发展, 智能硬件被越来越多的引入到设计教育的实践教学中。本文首先阐述了智能 硬件的特征以及设计生态模式的转变, 进而分析了智能硬件对设计教育的影响。最后, 结合实际的教学案例阐述了如何通过 4 种问题引导的模式来构建基于问题导向的智能硬件教学模式。

关键词：智能硬件；设计教育；教学模式

中图分类号：TP368.1 文献标志码：A

\section{1 引言}

现如今, 人类的产品承载了越来越多的信息化内容, 大量的数据也伴随产品的使用产生, 使得产品的 智能化具有了物质基础 [1]。这其中所应用到的互联网技术、传感器技术、情景感知算法等诸多的技术是 设计师拓展和实现设计概念的基础 [2]，其对设计教育的发展也有了较为深远的影响。本文具体从以下几 方面展开分析:

\section{2 智能硬件的特征}

这里所说的智能硬件既不同于电脑和智能手机, 也不同于机器人，是指以智能技术、互联网和大数据 技术为基础的软硬件结合的、拥有智能化功能的产品, 其主要包括以下两类: 一是被智能改造过的硬件, 例如智能家居设备、智能路由器、智能旅行箱等; 二是智能化的新硬件, 例如四轴飞行器和 $3 \mathrm{D}$ 打印机等 不含复杂机械的硬件。[3] 


\section{1 智能硬件的技术特征}

开源智能硬件得以迅速发展。首先应归功于两个因素。一是以 ARM 架构为基础的 GPU 计算芯片的快速 发展, 给智能硬件安了一颗具有强大计算能力的心脏 [4]; 二是物联网的建设思路, 为环境和各种产品添 加了强大的感知能力 [5]。这种计算能力和感知能力的增强。是智能硬件得以存在的基础。

\section{2 “开源精神” 让智能硬件的各种成本大大降低}

所谓的开源性是指大家会把自己所开发的智能硬件所有代码和细节都会公布在相关的论坛上，这样其 他人便可以在其基础上进一步开发和讨论。当前, 以 Arduino 和树莓派等为代表的开源硬件平台是学习智 能硬件的首选资源。这些平台一方面保持了较低的硬件成本, 可以让学生进行大胆尝试; 更为重要的一点 是开源硬件所崇尚的“开源精神”极大的拉低了智能硬件的学习成本。学习者在智能硬件的开发中不必自己 一个人从头到尾完成所有的开发。而是根据别人已经开源出来的各种代码、所使用的硬件模式基础之上进 一步开发自己的产品。这将大大提高学习和开发的效率。

\section{3 智能硬件打通了机械、电子和计算机学科之间的界限}

以 Arduino 为代表的各种开源硬件平台设计思路是先用各种电子化的传感器, 采集相关数据, 再通过 单片机的计算、分析和判断, 最终转化为机械化的控制输出 [6]。从而, 完成相关的功能需求。从这里我 们可以清晰的看到机械、电子和计算机三个学科之间的相互配合, 它们三个是一个完整的有机整体闭环。 通过智能硬件的开源平台的学习, 可以很自然的打通机械、电子和计算机学科的的界限, 提高学习的目的 性和效率, 为培养工业设计专业学生的技术素养与技术应用能力打下坚实的基础。

\section{3 设计教育之变}

我在英国进行访学的期间, 发现了一个很有趣的事情。当时访学所在的伦敦布鲁内尔大学, 其本科的 设计专业三年只有 18 门课程 [7]。无独有偶, 日本的千叶大学四年本科也只有 24 门课程。这与国内工业 设计专业动辄五十到六十门课程体系形成鲜明对比。这两所大学的课程设置思路可以被称之为以问题为导 向的教学 (problem-based learning, PBL), 即学习不再是基于严谨的学科分类知识体系进行教学, 而是 基于问题或者现象展开的教学模式。

\begin{tabular}{|c|c|}
\hline 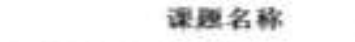 & 泟起设㬰 \\
\hline 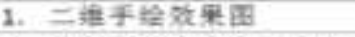 & 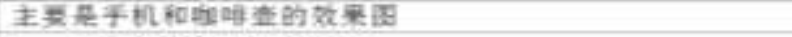 \\
\hline 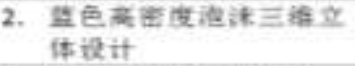 & 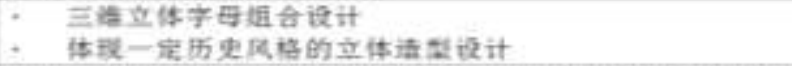 \\
\hline 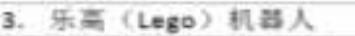 & 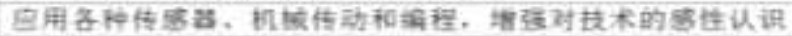 \\
\hline 4. 暊面设 it & 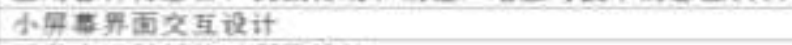 \\
\hline 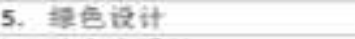 & 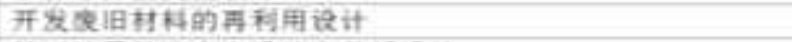 \\
\hline 6. D实实埌计 & 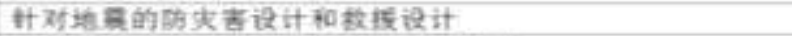 \\
\hline 7. 传鹤设 it & 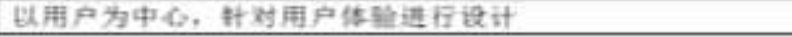 \\
\hline
\end{tabular}

这里我们通过仔细解剖一下伦敦布鲁内尔大学在本科一年级的一门课“Design Process ”( 设计过程), 来进一步说明基于问题导向的课程设计。这门课程作为设计专业的入门课程类似我国的 “设计概论”, 但 实际上课内容则要深得多也广的多, 反倒是像一个浓缩版的设计专业教育。其融合了我们的基础课、专业 基础课、专业课的 6-7 门课程的内容量。为了完成这么多的教学任务量, 整个课程设计了如表 1 所列举的 7 个设计课题。学生的学习就是围绕这 7 个课题展开的, 而不再是按照学科知识体系来按部就班的学习。 
通过上述分析，我们可以发现设计教育的理念在国外已有新的模式，呈现了不同的课程设置思路。我 们比较看重知识的完整性和系统性, 强调基础要打得牢, 所以在安排课程的时候会更尊重原有学科知识体 系。而布鲁内尔大学和千叶大学会更注重能力的培养, 强调学科知识要在能力的应用过程当中体现出来, 所以他们会主动打破学科的界限, 知识的学习围绕能力的增长而展开, 于是采用了很多基于问题导向的学 习模式。

\section{4 教学案例}

基于上述的分析, 我们认为智能硬件的相关课程从智能交互的角度被引入到工业设计教学当中是学科 发展的必然。一个新的学科被引入进来, 是原有知识体系的直接嫁接? 还是基于设计思维的有机融合?

为了回答这个问题我们选取了《设计基础》这门课来进行试验, 这是一门从基础课向专业课转换的过 渡课。并在两个学期先后尝试了两种教学模式: 一种是按照传统的教学模式, 先讲解 Arduino 的构造, 再 讲解如何用 Arduino 控制相关的传感器的知识 (称之为 A 模式。); 另一种是上来并不直接讲解 Arduino 的 构造, 只是从计算机原理的角度讲清楚 Arduino 是如何计算和控制传感器的, 大概 2 节课的时间, 然后就 抛出一个设计问题, 让学生思考 Arduino 可以解决其中的哪些环节。(称之为 B 模式。)。这两者的思路中 A 模式是按照原有学科的体系来安排课程的, $\mathrm{B}$ 模式是基于设计思维按照以问题为导向的教学方法来组织教 学的。

由于我们的学生是工科学生, 当采用模式 $\mathrm{A}$ 的时候, 学生没有表现出什么不适应。但当开始采用模式 $\mathrm{B}$ 的时候, 初始阶段学生出现了较大的不适应, 他们总是很担心智能硬件平台不能支持他们的设计方案。 于是, 我们给他们展示了一些用智能平台开发的比较有意思的案例, 并把一些开源硬件的论坛资源发给他 们。这样坚持了 3 次课以后, 学生开始进入状态了, 好多同学从相关论坛找到了技术原型, 他们的思考重 点慢慢又回到了设计方案本身。

另外一个有意思的点是, 模式 B 最终产品的复杂度要远远高于模式 $A$ 。这里的复杂度是指每个团队在 设计方案中所使用的传感器和各种零件平均数 (模式 A: 2.2 个, 模式 B: 4.8 个)。甚至出现两个团队因 追求过高的复杂度, 导致在课程最终演示时部分预定功能无法实现的问题。从这里我们能够看到学生不再 有对技术的恐惧, 反倒有着强烈的挑战意味。

作为号称 “信息黄埔” 的北邮学生, 其对于机械类的课程似乎有一种的天生的 “忽视” 潜意识, 他们 会觉得信息的时代将会是 APP 一类软件产品为主的时代。但在模式 B 下有几组对机械产生了浓厚兴趣, 并 开始回溯以前机械原理种中 “机械运动学” 的知识。课后他们感叹要是当初用这样的方法来学机械效率和 效果都会好很多。

\section{5 基于问题导向的智能硬件学习模式}

根据前面的分析, 我们采取了模式 B 的方案, 即通过提出问题来引导学生的进一步学习行为的智能硬 件学习模式（图 1) 的设计思路: 


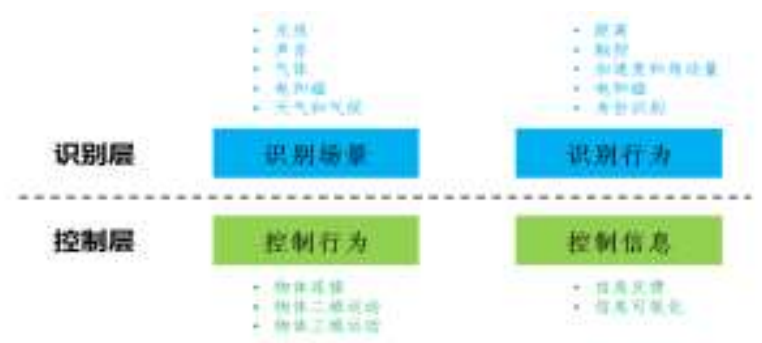

图 1 智能硬件学习模式图

\section{1 技术学习的思路}

首先我们从技术的角度我们将技术分为 “识别层” 和 “控制层” ，并进一步将识别层细化为 “识别场 景” 和 “识别行为” ; 控制层细化为 “控制行为” 和 “控制信息”。每一个细化层面都对应着相关的 “知 识点”, 学生的自主学习便是在这样的框架下, 结合开源论坛的各种设计原型来把这些知识点消化和理解 的。这样基本打破了原有知识体系的隔阂, 为知识的交叉奠定基础。

\section{2 问题设计的思路}

根据识别层和控制层的细化，我们可以得到四种排列组合: 识别场景+控制行为; 识别场景+控制信息; 识别行为+控制行为; 识别行为+控制信息。这四种组合就是学生提出问题和解决问题的模式。其中识别层 是输入、隐含着用户需求; 控制层是输出、是问题的解决方案。这样便将 “设计思维” 与技术的输入和输 出对应起来了, 实现了设计思维对问题的引导。

举例来说: 通过窗帘来识别光线 (识别场景) 以决定是否打开 (控制行为), 或者识别温度 (识别场 景) 来显示室外的气温 (控制信息); 通过座椅来识别人坐了多久 (识别行为) 来控制椅子面倾斜 (控制 行为）或者发出不舒服的声音（控制信息）以提醒人起身活动。

\section{6 结语}

综上所述, 这是我们对智能硬件对设计教育影响的思考。其主要落脚点还是希望打破学科之间的界限, 提升学生对机械、电子和计算机知识的综合运用能力, 为用设计思维来引导学生发现问题并创造性的解决 问题奠定坚实的基础。

\section{7 致谢}

本文系北京邮电大学教改项目 “基于 Processing 与 Arduino 的创新产品开发实践教学平台搭建与教 学模式研究”研究成果之一。

\section{参考文献:}

[1] 林泽航. 智能硬件发展趋势及创客运动研究 [A]. 数字技术与应用, 2016， 37 卷 (8 期)： 72 .

[2] 安晖, 温晓君. 中国智能硬件产业发展现状 [J]。互联网经济, 2015（Z2）：35-39.

[3] 吴平. 数字化时代智能硬件特点分析及设计思路探讨 [A]. 包装工程, 2016， 37 卷 (8 期)： 25-26.

[4] 李世国. 物联网时代的智慧型物品探析 $[\mathrm{A}]$. 包装工程, 2010， 31 卷（4 期)： 50

[5] 杨楠. 物联网环境下智能产品原型设计研究. 包装工程, 2014， 35 卷 (6 期)： 55

[6] 付久强. Arduino 平台智能硬件设计研究 [A]. 包装工程. 2015， 36 卷 (10 期)： $76-77$

[7] 布鲁内尔大学产品设计专业 http://www. brunel. ac. uk/study/undergraduate/product-design-bsc

\section{References}

[1] Lin Ze-hang:" Research on intelligent hardware trend and maker movement"[J]. Digital technology and Application. Vol.37(2016), No.9, p.52-55 (in Chinese) 
[2] An Hui, Wen Xiao-jun:"Development status of intelligent hardware industry in China"[J]. Internet economy. VolZ2(2015), p.35-39(in Chinese)

[3] Li Shi-guo:" Study on Intelligent Things in the Internet of Things". Packaging Engineering. Vol.31(2010), No.4, p.50 (in Chinese)

[4] Wu Ping:" Characteristics Analysis and Design Thinking of Intelligent Hardware in the Digital Age". Packaging Engineering. Vol.37(2016), No.8, p.24-25 (in Chinese)

[5] Yang Nan:" Intelligent Product Prototype Design in the Internet of Things Environment". Packaging Engineering. Vol.35(2014), No.6, p.24-25 (in Chinese)

[6] Fu Jiu-qiang:" Intelligent Hardware Design Based on the Arduino Platform". Packaging Engineering. Vol.36(2015), No.10, p.76-77 (in Chinese)

[7] http://www.brunel.ac.uk/study/undergraduate/product-design-bsc 